\title{
Mejorando la pesquisa de depresión posparto a través de un instrumento de tamizaje, la escala de depresión posparto de Edimburgo
}

\author{
Carla Castañón $\mathbf{S}^{1}$, Javier Pinto $\mathbf{L}^{2}$. \\ Use fo the Edinburgh postnatal \\ depression scale to detect \\ postpartum depression
}

Background: Postpartum depression is a serious, common, treatable condition, with broad repercussions in mother and family life, but frequently under diagnosed. A screening tool, the Edinburgh Postnatal Depression Scale (EPDS) could improve the detection rate of depressive symptoms. Aim: To assess the efficacy of EPDS in the detection rates for depressive symptoms in the postpartum period. To evaluate the feasibility of routine use of this screening scale in a Family Medicine Center. Material and methods: The medical records of women attended during 2002, were reviewed to detect the registry of depressive symptoms or request for psychiatric consultations for depression. During 2003, the EPDS was applied to a sample of 110 women. To confirm the postpartum depression diagnosed using the instrument, a structured psychiatric interview was administered to these women. Results: The medical records of 110 women aged $24 \pm 7$ years were reviewed and the instrument was applied to 110 women aged $26 \pm 7$ years. In the first group, the spontaneous detection rate for depressive symptoms was $4.6 \%(5 / 110)$. In $26.4 \%$ of women in whom the instrument was applied, depressive symptoms were found and $15 \%$ had a postpartum depression. The application rate of the scale was $88 \%$ and the rate of acceptance by mothers was $100 \%$. Conclusions: The EPDS improves significantly the detection rates of postpartum depressive symptoms, and it is well accepted by health professionals and mothers (Rev Méd Chile 2008; 136: 851-8).

(Key words: Depression, postpartum; Psychiatry; Questionnaire)

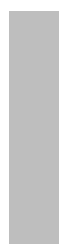

Recibido el 27 de noviembre, 2007. Aceptado el 14 de marzo, 2008.

Este estudio contó con el apoyo financiero del Departamento de Medicina Familiar de la Pontificia Universidad Católica de Chile, pero el diseño del estudio y el análisis de los resultados se realizaron en forma independiente de este Departamento.

${ }^{1}$ Departamento de Medicina Familiar, Pontificia Universidad Católica de Chile. Santiago de Chile. ${ }^{2}$ Departamento de Psiquiatría, Pontificia Universidad Católica de Chile. Santiago de Chile.

Correspondencia a: Dra. Carla Castañón S. Edificio Decanato, Departamento Medicina Familiar, Pontificia Universidad Católica de Chile. Lira 44, Santiago. Dirección particular: Las Araucarias 7834, Santiago. Fax: 5573315.

E mail: ccastano@puc.cl 
$\mathrm{U}$ no de los principales factores protectores para un adecuado desarrollo de los niños lo constituye la existencia de un buen vínculo madre-hijo. Sin embargo, es precisamente en los inicios de esta relación, el período posparto, cuando se presentan con mayor frecuencia síntomas depresivos, que de acuerdo a su severidad comprometen en mayor o menor grado las capacidades maternas y amenazan el normal desarrollo de dicha relación ${ }^{1}$.

La depresión posparto (DPP) es una condición seria, frecuente, con repercusiones no sólo a corto plazo. La mayoría de las mujeres padece la enfermedad por más de 6 meses, y si no son tratadas, 25\% puede persistir con síntomas aun al año de evolución ${ }^{2}$. Además, tendrán un mayor riesgo de recurrencia de estos episodios en embarazos subsecuentes (1:3 a 1:4), incluso más seve$\operatorname{ros}^{3}$.

En los niños, el trastorno en la relación madrehijo tendrá un impacto negativo, e inversamente proporcional a la duración del episodio, en su desarrollo emocional y cognitivo global (verbal, motor, perceptivo), exacerbando incluso los efectos de variables asociadas con menor desarrollo mental y del lenguaje, tales como un menor nivel socioeconómico ${ }^{4}$. Los hijos de madres con DPP desarrollan relaciones de apego inseguro con una frecuencia significativamente mayor que los hijos de madres sin depresión, una mayor frecuencia de trastornos conductuales, de accidentes, de ingresos hospitalarios y un mayor riesgo de maltrato infantil ${ }^{4-8}$.

De acuerdo al Diagnostic and Statistical Manual of Mental Disorders, cuarta edición (DSMIV), un episodio de depresión se considera de presentación posparto si comienza dentro de las 4 semanas posparto, con un marco de tiempo para efectos de investigación de hasta 3 meses posparto $^{9}$. La prevalencia descrita internacionalmente fluctúa entre $10 \%$ y $20 \% 10-15$, siendo mayor en niveles socioeconómicos más bajos y en madres adolescentes ${ }^{10,16}$.

Estudios realizados en Suecia y Estados Unidos de Norteamérica (EE.UU.) describen tasas de detección espontánea de síntomas depresivos en el período posparto de $2 \%$ y $6,3 \%$, respectivamente ${ }^{17,18}$. Sin embargo, al utilizar un instrumento de tamizaje simple y rápido de contestar, la Escala de Depresión Postnatal de Edimburgo (EDPE), estas tasas aumentaron a 14,5\% cuando se utilizó un punto de corte $12 / 13$ y 35,4\% al utilizar un punto de corte 9/10, respectivamente. En Chile, utilizando la misma herramienta, la prevalencia de sintomatología depresiva en el período posparto fue de $41,3 \%$ en el nivel socioeconómico bajo, 33,5\% en nivel socioeconómico medio y $27,7 \%$ en el nivel socioeconómico alto ${ }^{19}$.

Considerando que la derivación para consulta psiquiátrica puede no ser expedita, es posible realizar la evaluación de estas pacientes a través de una entrevista psiquiátrica estructurada ${ }^{20,21}$, a cargo de médicos capacitados, quienes podrán iniciar el tratamiento oportunamente, y derivar sólo a aquellas pacientes con sintomatología más severa o con comorbilidad psiquiátrica.

El propósito de este estudio fue evaluar la eficacia de la EDPE en mejorar la tasa de detección de síntomas depresivos en el período posparto, y la viabilidad de implementar rutinariamente esta escala de tamizaje en un Centro de Salud Familiar.

\section{MATERIAL Y MÉTODO}

El estudio se desarrolló en el Centro de Salud Familiar El Roble, comuna de La Pintana, Santiago de Chile. Se incluyó en forma correlativa a las madres que asistían a supervisión de salud de sus hijos desde el primer mes de vida, excluyendo aquellas con menos de 4 años de escolaridad 0 que hubiesen recibido tratamiento antidepresivo en los últimos 6 meses.

Instrumento de tamizaje. La Escala de Depresión Postnatal de Edimburgo es una encuesta autoadministrada, desarrollada para la detección de síntomas depresivos en mujeres que hayan dado a luz recientemente. Consta de 10 preguntas de selección múltiple, con 4 alternativas cada una, puntuada de 0 a 3 de acuerdo a la severidad creciente de los síntomas. (Anexo 1).

Ha sido validada en diversos países y culturas, incluyendo Chile ${ }^{22}$, logrando su mayor sensibilidad con un punto de corte $9 / 10^{23}$, constituyendo el punto de corte más apropiado para estudios de tamizaje ${ }^{24}$.

Se aplicó la versión en español utilizada en una de las validaciones chilenas ${ }^{22}$. 


\section{ANEXO 1 - HERRAMIENTA DE TAMIZAJE}

Escala de Edimburgo. Versión validada por Jadresic E, Araya R, Jara C. (J Psychosom Obstet Gynecol 1995; 16: 187-91)

\section{ESCALA DE EDIMBURGO}

Como usted hace poco tuvo un bebé, nos gustaría saber cómo se ha estado sintiendo. Por favor SUBRAYE la respuesta que más se acerca a cómo se ha sentido en los últimos 7 días.

En los últimos 7 días:

1) He sido capaz de reírme y ver el lado divertido de las cosas:

tanto como siempre

no tanto ahora

mucho menos ahora

no, nada

2) He disfrutado mirar hacia delante:

tanto como siempre

menos que antes

mucho menos que antes

casi nada

3) Cuando las cosas han salido mal, me he culpado a mí misma innecesariamente:

sí, la mayor parte del tiempo

sí, a veces

no con mucha frecuencia

no, nunca

4) He estado nerviosa o inquieta sin tener motivo:

no, nunca

casi nunca

sí, a veces

sí, con mucha frecuencia

5) He sentido miedo o he estado asustadiza sin tener motivo:

sí, bastante

sí, a veces

no, no mucho

no, nunca
6) Las cosas me han estado abrumando:

sí, la mayor parte del tiempo no he podido hacer las cosas en absoluto

sí, a veces no he podido hacer las cosas tan bien como siempre

no, la mayor parte del tiempo he hecho las cosas bastante bien

no, he estado haciendo las cosas tan bien como siempre

7) Me he sentido tan desdichada que he tenido dificultades para dormir:

sí, la mayor parte del tiempo

sí, a veces

no con mucha frecuencia

no, nunca

8) Me he sentido triste o desgraciada:

sí, la mayor parte del tiempo

sí, bastante a menudo

no con mucha frecuencia

no, nunca

9) Me he sentido tan desdichada que he estado

llorando:

sí, la mayor parte del tiempo

sí, bastante a menudo

sólo ocasionalmente

no, nunca

10) Se me ha ocurrido la idea de hacerme daño:

sí, bastante a menudo

a veces

casi nunca

nunca
Diseño. Para evaluar la eficacia de la escala de tamizaje en mejorar la detección de síntomas depresivos, utilizando metodología cuantitativa, se realizó un estudio con diseño antesdespués, comparando 2 muestras de madres puérperas, en períodos equivalentes de 2 años consecutivos.
El tamaño muestral fue calculado con el programa S-PLUS 2000, utilizando tasas de detección hipotéticas de síntomas depresivos, basándose en la literatura existente, de $5 \%$ antes del tamizaje y de $20 \%$ después del tamizaje, $\alpha=.05$, poder $=.80$, a lo cual se agregó $20 \%$ de la muestra calculada anticipando posibles pérdidas en el 
seguimiento. De este modo el tamaño de cada grupo fue de 110 madres.

Para determinar la prevalencia de depresión posparto en el grupo intervenido, se aplicó el supuesto de que la EDPE tiene una sensibilidad de $100 \%$ con el punto de corte utilizado, de acuerdo a lo reportado en diversos estudios.

Implementación de la escala. Se realizó un proceso de capacitación en la forma de aplicación (auto-administrada) y de interpretación (puntaje) de la EDPE, y en el sistema de derivación para la entrevista psiquiátrica. Las madres podían optar voluntariamente a responder o no la escala, la cual se aplicó, previo consentimiento de ellas, durante la supervisión de salud sus hijos, entre las 4 y 12 semanas posparto.

Para determinar la viabilidad de la aplicación rutinaria de la EDPE, se calculó la tasa de aplicación de la escala por parte de los profesionales y la tasa de aceptabilidad de la escala por parte de las madres.

Diagnóstico de depresión mayor en el posparto y tratamiento. Las madres que obtuvieron puntaje mayor o igual a 10 fueron derivadas para la realización de la entrevista psiquiátrica.

Para el diagnóstico de depresión mayor, y considerando la alta demanda hacia el nivel secundario por atención en Salud Mental, se capacitó a residentes de Medicina Familiar, con formación y experiencia en dicha área, en una entrevista semiestructurada para los trastornos del eje I del DSMIV (SCID-I) adaptada al español, y aplicada previamente en nuestro país, utilizando las secciones de antecedentes generales y trastornos afectivos de su versión clínica. Esta capacitación fue realizada por un psiquiatra experimentado en la aplicación de la entrevista, y a través de análisis de entrevistas grabadas, role-playing (paciente simulado), y evaluación con pacientes.

El tratamiento farmacológico de las pacientes podía ser iniciado por el mismo médico, definiéndose como criterios de derivación al nivel secundario: ideación suicida u homicida, presencia de síntomas psicóticos, funcionamiento global severamente comprometido, comorbilidad con abuso de sustancias y mala respuesta al tratamiento indicado.

Recolección de datos. Los datos sociodemográficos (edad, paridad, escolaridad, estado civil, fecha de parto) fueron recolectados desde los registros clínicos de la madre y del hijo.

Para determinar la tasa de detección de síntomas depresivos en el grupo pre-intervención, se revisó las fichas clínicas de las madres y de los hijos en busca del registro de estos síntomas o de derivaciones por esta causa (mayo a julio de 2002).

La presencia de síntomas depresivos en el grupo intervenido se definió como un puntaje de 10 o más en la EDPE.

Análisis de los datos. Las diferencias en las variables sociodemográficas y en las tasas de síntomas depresivos fueron evaluadas con t-test para 2 muestras, test exacto de Fisher o test de Chicuadrado según correspondiera, con los respectivos IC.

La significancia estadística se definió en $<0,05$ o con IC 95\% que no incluyera 1.

\section{Resultados}

Comparación de los grupos. Para las variables comparadas (edad, estado civil, paridad) y su relación con la presencia o no de síntomas depresivos, se encontró diferencia significativa entre los grupos solamente en los promedios de edad respectivos $(p=0,036)$. Sin embargo, al realizar el cruce entre estas variables y la presencia o no de síntomas depresivos, la diferencia no fue estadísticamente significativa. Tablas 1 y 2.

Capacitación en escala y entrevista psiquiátrica. En abril y mayo de 2003 se capacitó a 85\% de los profesionales involucrados y el $15 \%$ restante fue capacitado individualmente. Se capacitó también en forma individual a aquellos profesionales que comenzaron a trabajar en el Centro de Salud después de mayo de 2003.

En mayo de 2003 se capacitó a 3 residentes de Medicina Familiar en la entrevista psiquiátrica (SCID-I), quienes cumplieron con $100 \%$ de las actividades que incluía la capacitación.

Aceptación de la escala. En relación a la implementación de la escala, la tasa de aplicación de la EDPE por parte de los profesionales fue de $88 \%$. La escala fue aceptada por $100 \%$ de las madres. 
Tabla 1. Comparación de características sociodemográficas de los grupos

\begin{tabular}{|lccc|}
\hline & $\begin{array}{c}\text { Grupo sin } \\
\text { intervención } \\
(\mathrm{n})\end{array}$ & $\begin{array}{c}\text { Grupo con } \\
\text { intervención } \\
(\mathrm{n})\end{array}$ & Valor $\mathrm{p}$ \\
\hline $\begin{array}{l}\text { Edad (años) } \\
\text { Promedio } \pm \text { DS }\end{array}$ & $23,9 \pm 6,5(134)$ & $25,6 \pm 6,9(139)$ & $0,036(1)$ \\
& & & \\
Estado civil (\%) & $66,4(89)$ & $73,2(93)$ & $0,165(2)$ \\
Casada/convive & $9,7(13)$ & $4,0(5)$ & \\
Con pareja/no conviven & $23,9(32)$ & $22,8(29)$ & \\
Sin pareja & & & \\
& & $40,2(51)$ & \\
Paridad (\%) & $50,0(67)$ & $41,7(53)$ & \\
Primípara & $38,1(51)$ & $18,1(23)$ & \\
$>1$ < hijos & $11,9(16)$ & & \\
$>3$ hijos & & & \\
\hline
\end{tabular}

(1) t-test

(2) chi-cuadrado

Tabla 2. Presencia de síntomas depresivos según variables sociodemográficas

\begin{tabular}{|lccc|}
\hline & $\begin{array}{c}\text { Escala Edimburgo } \\
\text { Puntaje } \geq 10 \\
(\mathrm{n})\end{array}$ & $\begin{array}{c}\text { Escala Edimburgo } \\
\text { Puntaje }<10 \\
(\mathrm{n})\end{array}$ & Valor $\mathrm{p}$ \\
\hline $\begin{array}{l}\text { Edad promedio + DS } \\
\text { Estado civil (\%) }\end{array}$ & $26,0 \pm 7,5(45)$ & $25,4 \pm 6,6(74)$ & $0,631(1)$ \\
$\quad$ Casada/convive & $71,1(27)$ & $71,0(49)$ & $0,228(2)$ \\
Con pareja/no conviven & $-(0)$ & $7,3(5)$ & \\
Sin pareja & $29,0(11)$ & $21,7(15)$ & \\
Paridad (\%) & $36,8(14)$ & $42,0(29)$ & $0,863(3)$ \\
Primípara & $44,7(17)$ & $42,0(29)$ & \\
$>1<3$ hijos & $18,4(7)$ & $16,0(11)$ & \\
$>3$ hijos & & & \\
\hline
\end{tabular}

(1) t-test

(2) test exacto de Fisher

(3) chi-cuadrado

Entrevista psiquiátrica. La latencia entre la detección de síntomas depresivos con la EDPE y la entrevista psiquiátrica fue variable, oscilando entre 1 y 3 semanas. Se debió priorizar, de acuerdo a la intensidad de los síntomas, debido al balance negativo entre las horas de atención disponibles para la entrevista y el número de pacientes que la requerían.
Detección de síntomas depresivos. La tasa de síntomas depresivos reportada en los registros clínicos de madres y niños en el grupo preintervención fue de 4,6\% (5 de 110) (Figura 1).

La tasa de síntomas depresivos en el grupo de pacientes a las cuales se les aplicó la EDPE fue de 26,4\% (29 de 110) (Figura 2). 
La diferencia entre ambos grupos fue estadísticamente significativa (chi-cuadrado $=44,6, \mathrm{df}=1, \mathrm{p}$ $<0,001)$.

Frecuencia de depresión mayor en el posparto. Se realizó entrevista psiquiátrica a $76 \%(\mathrm{n}=22)$ de las pacientes con puntaje mayor 0 igual a 10 en la EDPE (las 7 restantes no asistieron a dicha entrevista y 2 de ellas no pudieron ser contactadas posteriormente).

De las pacientes entrevistadas, $55 \%$ ( $\mathrm{n}=16$ ) cumplía criterios para el diagnóstico de depresión mayor según DSM-IV, resultando una frecuencia de depresión posparto de $15 \%$, en un análisis con intención de tratar (Figura 2).

Derivación a nivel secundario. Se derivó al nivel secundario (COSAM de la comuna) a 19\% de las pacientes con depresión posparto (3 de 16). La mayoría de las pacientes con este diagnóstico continuó sus controles en el Centro de Salud, iniciándose tratamiento farmacológico y controles en el nivel primario en 9 de ellas. En las pacientes derivadas igualmente se inició tratamiento farmacológico.

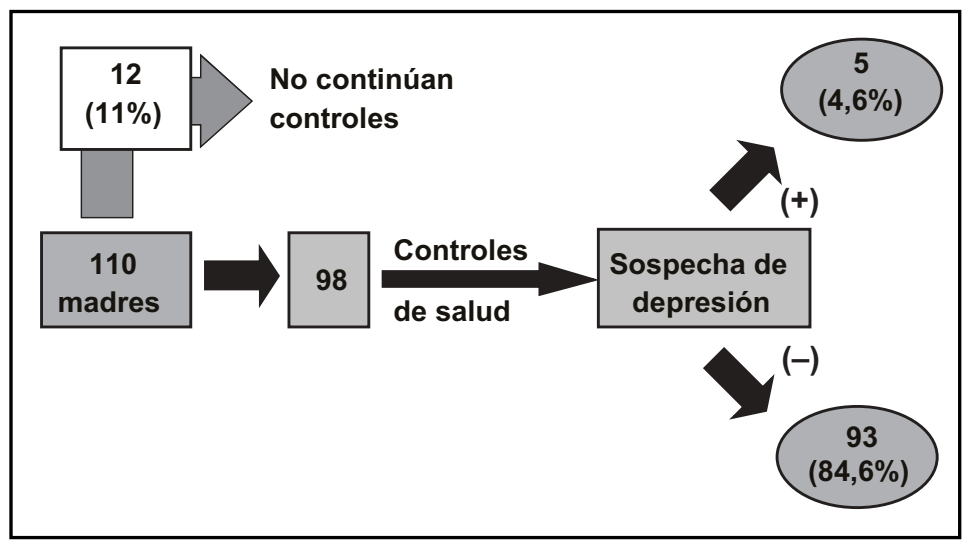

Figura 1. Detección espontánea de síntomas depresivos posparto.

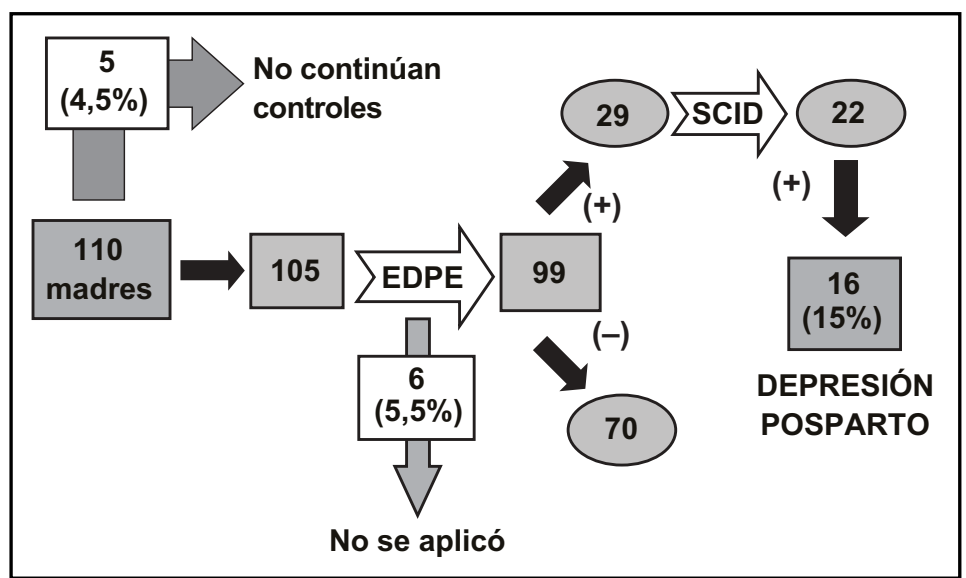

Figura 2. Detección de síntomas depresivos a través de tamizaje y certificación de depresión posparto.

\section{Discusión}

Los resultados de este estudio son concordantes con estudios previos en relación al aumento significativo de las tasas de detección de síntomas depresivos cuando se utiliza una herramienta de tamizaje como la Escala de Depresión Postnatal de Edimburgo ${ }^{7,8}$.

Consciente de las limitaciones que un estudio de tipo antes-después puede tener, en términos de subestimar factores que pudiesen modificar los resultados, la diferencia altamente significativa encontrada en el estudio permite suponer que ésta se deba principalmente a la implementación de la escala de tamizaje. Además, dicha escala permite también identificar indirectamente otros factores estresantes para la madre, que se manifiesten en un mayor puntaje, y sobre los cuales es posible intervenir o brindar apoyo, como son la presencia de conflictos maritales, de riesgo de violencia intrafamiliar o maltrato infantil.

La alta motivación por parte del equipo de salud para la implementación rutinaria de la escala de tamizaje, expresada en el alto porcentaje de aplicación de ésta (muy superior al reportado en otros estudios), y la excelente aceptación por parte de las madres para responder la escala, hacen atractivo incluir esta estrategia como una actividad 
rutinaria en los controles de salud del niño y su madre. En el pequeño grupo en que no se aplicó la escala, no se indagó en las causas de ello.

Una fortaleza de este estudio es que fue desarrollado en un centro de salud público, sistema que atiende a la mayor parte de la población, lo que permitió enfrentar los desafíos de una realidad más compleja en términos de tiempo de atención por paciente, continuidad de la atención y acceso a especialistas. Como ventaja adicional, está el incorporar médicos de atención primaria en el diagnóstico y tratamiento de la depresión posparto. Si bien el diagnóstico se realizó mediante una entrevista psiquiátrica estructurada, que requería de $30 \mathrm{~min}$ para su aplicación, esto fue requerido sólo como una forma de estandarización para el estudio.

En la práctica cotidiana, profesionales con formación en el diagnóstico y tratamiento de la patología depresiva pueden llevar a cabo ambos procesos durante la entrevista clínica habitual, estableciendo previamente criterios de derivación al especialista, lo que permite agilizar el abordaje de las madres con síntomas depresivos y evitar sobrecargar al nivel secundario de atención.

Una modificación interesante a la estrategia utilizada puede ser retrasar la aplicación de la escala hasta alrededor de las 8 semanas posparto, fundamentado en que dicho período representa el máximo de prevalencia de la depresión posparto, y además ya han disminuido los síntomas atribuibles a un período adaptativo normal. Esto permitiría una mayor especificidad y un mayor valor predictivo positivo, considerando que casi un tercio de las pacientes a las que se realizó la entrevista psiquiátrica no cumplía criterios de depresión mayor. Otra alternativa es aplicar la escala entre las 4 y 6 semanas posparto, derivando inmediatamente a evaluación médica sólo a quienes obtienen un puntaje mayor o igual a 13, y repetirla un mes después a aquellas madres con puntaje entre $10 \mathrm{y}$ 13, salvo que presenten antecedentes de depresión previa o ideación suicida. Esto se fundamenta en estudios de validación que han encontrado una sensibilidad aceptable de la escala al utilizar un punto de corte 12/13, y a que la mayoría de los falsos positivos de la escala se presentaron en el grupo con puntaje entre 10 y 12 .

Actualmente, la EDPE se administra en forma universal en la comuna de La Pintana, a las 8 semanas posparto, manteniendo el punto de corte 9/10.

Las proyecciones de este estudio guardan relación con la implementación de esta estrategia en otros centros de salud, tanto públicos como privados, la posibilidad de implementar estrategias adicionales de detección de síntomas depresivos, por ejemplo durante el embarazo (hasta 50\% de los casos de depresión posparto pueden ser la continuación de episodios depresivos que ocurren durante 0 antes del embarazo) y la posibilidad de aumentar las alternativas de tratamiento hacia intervenciones no farmacológicas, individuales 0 grupales, que pudieran cubrir mejor las necesidades de apoyo que necesitan estas madres y sus hijos.

Asimismo, la incorporación de la depresión dentro de las patologías con garantías explícitas en salud (GES) puede contribuir a optimizar el tratamiento no farmacológico y farmacológico de esta condición, y hacer más expedita la derivación a especialista en los casos que lo ameriten.

\section{CONCLUSIONES}

La depresión posparto es una condición seria, frecuente y tratable, que tiene repercusiones a corto y largo plazo en la salud del niño y la madre.

Este tipo de depresión es habitualmente subdiagnosticada por los profesionales de la salud, por lo que se requiere implementar estrategias de tamizaje para aumentar la tasa de detección.

La Escala de Depresión Postnatal de Edimburgo es una herramienta fácil de aplicar, bien aceptada por los profesionales de salud y por las madres, y que permite aumentar significativamente la tasa de detección de síntomas depresivos.

La depresión posparto puede ser diagnosticada y tratada en Centros de Atención Primaria, derivando al nivel secundario sólo casos seleccionados debido a su mayor severidad.

\section{Agradecimientos}

Expresamos nuestros agradecimientos a las madres que aceptaron participar en este estudio, al personal de salud del Centro de Salud Familiar El Roble y al Departamento de Medicina Familiar de la Pontificia Universidad Católica de Chile. 


\section{REFERENCIAS}

1. Stern DN. La constelación maternal. Ediciones Piadós Ibérica, 1995, Barcelona, España.

2. Gregoire AJ, Kumar R, Everitt B, Henderson AF, Studd JW. Transdermal oestrogen for treatment of severe postnatal depression. Lancet 1996; 347 (9006): 9303.

3. Hamilton JA. Postpartum psychiatric syndromes. Psychiatr Clin North Am 1989; 11: 341-50.

4. COOPER P, MurRaY L Fortnightly review: Postnatal depresssion. BMJ 1998; 316: 1884-6.

5. Teti DM, Geifand CM, Messinger DS, Isabeila R Maternal depression and the quality of early attachment: an examination of infants, preschoolers, an their mothers. Devel Psychology 1995; 31: 364-76.

6. Cogill S, Capian H, Aiexandra H, Robson KM, Kumar R. Impact of postnatal depression on cognitive development in young children. BMJ 1986; 292: 1165-7.

7. MurRay L Postnatal depression and infant development. BMJ 1991; 302: 978-9.

8. Murray L, Sinclair D, Cooper P, Ducournau P, Turner P. The socioemotional development of 5 years olds with postnatally depressed mothers. J Child Psychol Psychiatry 1999; 40: 1259-71.

9. Wisner KL, Parry BL, Piontek CM. Postpartum depression. N Engl J Med 2001; 347: 194-9.

10. Jadresic E, Jara C, Miranda M, Arrau B, Araya R. Trastornos emocionales en el embarazo y el puerperio: estudio prospectivo de 108 mujeres. Rev Chil Neuro-Psiquiat 1992; 30: 99-106.

11. Alvarado R, Rojas M, Monardes J, Neves E, Olea E, Perucca E, Vera A. Cuadros depresivos en el postparto $\mathrm{y}$ variables asociadas en una cohorte de 125 mujeres embarazadas. Rev Psiquiatr 1992; IX: 1168-76.

12. Cox JL, Murray D, Chapman G. A controlled study on the onset, duration and prevalence of postnatal depression. Br J Psychiatry 1993; 163: 27-31.

13. JosefsSon A, Berg G, Nordin C, Sydsjo G. Prevalence of depressive symptoms in late pregnancy and postpartum. Acta Obstet Gynecol Scand 2001; 80: 251-5.

14. Stowe ZN, Nemeroff CB. Women at risk for postpartum-onset depression. Am J Obstet Gynecol 1995; 173: 639-45.

15. Chaudron LH. Postpartum depression: what pediatricians need to know. Pediatrics in review 2003; 24: 154-60.

16. Troutman BR, Cutrona CE. Nonpsychotic postpartum depression among adolescent mothers. J Abnormal Psychol 1990; 99: 69-78.

17. Bagedaht-Strindlund M, Monsen Börjesson K. Postnatal depression; a hidden illness. Acta Psychiatr Scand 1998; 98: 272-5.

18. Evins GG, Theofrastous JP, Galvin SL Postpartum depression: A comparison of screening and routine clinical evaluation. Am J Obstet Gynecol 2000; 182: 1080-2.

19. Jadresic E, Araya R. Prevalencia de depresión postparto y factores asociados en Santiago, Chile. Rev Méd Chile 1995; 123: 694-9.

20. Sheehan DV, Lecrubier Y, Sheehan KH, Amorim P, Janavs J, Weiler E et al. The Mini-International Neuropsychiatric Interview (M.I.N.I.): the development and validation of a structured diagnostic psychiatric interview for DSM-IV and ICD-10. J Clin Psychiatry 1998; 59 Suppl 20: 22-33.

21. Spitzer RL, Wiwiams JB, Gibbon M, First MB. The Structured Clinical Interview for DSM-III-R (SCID). Arch Gen Psychiatry 1992; 49: 624-9.

22. JADRESIC E, ARAYA R, Jara C. Validation of the Edinburgh postnatal depression scale (EPDS) in Chilean postpartum women. J Psychosom Obstet Gynecol 1995; 16: 187-91.

23. Eberhard-Gran M, Eskild A, Tambs K, Opjordsmoen $S$, SAMuelsen SO. Review of validation studies of the Edinburgh Postnatal Depression Scale. Acta Psychiatr Scand 2001; 104: 243-9.

24. Murray L, Carothers A. The validation of the Edinburgh Post-natal Depression Scale on a community sample. Br J Psychiatry 1990; 157: 288-90. 